\title{
Human Platelet Aggregation Is Initiated by Peripheral Blood Mononuclear Cells Exposed to Bacterial Lipopolysaccharide In Vitro
}

\author{
Bradford S. Schwartz and Martha C. Monroe \\ Departments of Medicine and Human Oncology, University of Wisconsin, Madison, Wisconsin 53706
}

\begin{abstract}
Platelet consumption is a prominent feature of disseminated intravascular coagulation. We investigated whether monocyte procoagulant activity (PCA) might play a role in platelet consumption associated with gram-negative septicemia. Human mononuclear cells exposed in vitro to lipopolysaccharide demonstrated parallel dose-dependent increases in PCA and ability to induce platelet aggregation. Induction of platelet aggregation required the generation of thrombin dependent on coagulation Factors VII, X, and II, and calcium. This is consistent with monocyte tissue factor initiating thrombin generation. A specific monoclonal antimonocyte antibody was used to identify monocytes via indirect immunofluorescence, and demonstrated that all monocytes were included in platelet aggregates. Mononuclear cells that did not express PCA did not induce platelet aggregation and monocytes were not surrounded by platelet clumps. These data suggest that monocytes induced to express tissue factor on their surface may be important mediators of endotoxin-induced platelet, as well as fibrinogen, consumption.
\end{abstract}

\section{Introduction}

Thrombocytopenia is a consistent laboratory finding in patients with disseminated intravascular coagulation (DIC), ${ }^{1}$ which is a process also marked by consumption of fibrinogen (1). DIC can be caused by a number of processes, with septicemia due to gram-negative bacteria a frequent triggering event (2). Lipopolysaccharide (LPS) from endotoxin of gram-negative bacteria is believed to play a prominent causative role in such cases of DIC (3). A proposed mechanism for this is the induction of procoagulant activity (PCA) among peripheral blood mononuclear cells (PBM) after exposure to LPS (4). The PCA is expressed by monocytes, which require instructions from LPSstimulated $T$ lymphocytes to manifest maximum PCA $(5,6)$. Experimental evidence suggests mononuclear cell PCA has a causative role in DIC. Rendering an experimental animal leukopenic interferes with endotoxin-induced DIC (7). Transfusing a leukopenic animal with leukocytes restores the ability of endotoxin to induce fibrinogen consumption (8). Likewise, infusing an experimental animal with leukocytes that have been exposed

Received for publication 13 January 1986 and in revised form 24 July 1986.

1. Abbreviations used in this paper: DIC, disseminated intravascular coagulation; FITC, fluorescein isothiocyanate; LPS, lipopolysaccharide; PBM, peripheral blood mononuclear cells; PCA, procoagulant activity; TBS, tris-buffered saline.

J. Clin. Invest.

(c) The American Society for Clinical Investigation, Inc.

0021-9738/86/11/1136/06 \$1.00

Volume 78, November 1986, 1136-1141 to LPS in vitro, and that express procoagulant, results in DIC (9). Monocytes have been shown to be the cells expressing PCA among leukocytes (10).

These studies, however, have not addressed the mechanism of thrombocytopenia, a prominent part of the DIC. Indeed laboratory evidence of DIC can be found in almost all septic patients with a platelet count $<50,000 / \mu l(11)$. Endotoxin damages platelets (12), and some investigators have felt this to be the primary mechanism whereby endotoxin induces thrombocytopenia and DIC (13). However, thrombocytopenic animals demonstrate nearly normal rates of endotoxin-induced fibrin deposition in organs (14). We investigated the possibility that PBM exposed to LPS might initiate platelet aggregation. We felt it reasonable that the thrombin generated at the surface of monocytes as a result of tissue factor expression would cause platelet aggregation as well as clotting of fibrinogen, giving a unified mechanism of platelet and fibrinogen consumption.

\section{Methods}

Cell isolation and culture. Mononuclear cells were isolated from the peripheral blood of fasting healthy donors. Blood was drawn into a plastic syringe without anticoagulant and added immediately to a sterilized glass Erlenmeyer flask containing 155 -mm glass beads. The blood was then defibrinated, and the mononuclear cells subsequently isolated by FicollHypaque centrifugation $(1.077 \mathrm{~g} / \mathrm{ml}$, Sigma Chemical Company, St. Louis, MO) at $1,400 \mathrm{~g}$ and $22^{\circ} \mathrm{C}$ for $12 \mathrm{~min}$. This method yielded mononuclear cells completely free of platelet contamination as assessed by immunofluorescence for thrombospondin (15). Mononuclear cells were then washed twice with Hanks' balanced salt solution, resuspended in RPMI 1640 (Gibco, Grand Island, NY) containing $100 \mathrm{U} / \mathrm{ml}$ penicillin, $50 \mu \mathrm{g} / \mathrm{ml}$ streptomycin, $2 \mathrm{mM}$ Hepes (Gibco) $\mathrm{pH} 7.4$, and $10 \%$ heatinactivated fetal calf serum (FCS, Sterile Systems, Logan, UT). These PBM contained an average of $80 \%$ lymphocytes, $20 \%$ monocytes, and $<1 \%$ polymorphonuclear leukocytes as assessed by Wright's stain. Cell viability was $>99 \%$ as assessed by eosin exclusion. All media were prepared with pyrogen-free water and stored in acid-washed, high-temperature-baked glassware. PBM were cultured at $1 \times 10^{6}$ cells $/ \mathrm{ml}$ in $12 \times 75$ $\mathrm{mm}$ sterile polypropylene tubes (Falcon Labware, Oxnard, CA) at $37^{\circ} \mathrm{C}$, $5 \% \mathrm{CO}_{2}$, high humidity for the times indicated in the text. LPS (Escherichia coli B4:0111, Difco Laboratories, Detroit, MI) was added to cultures as indicated in the text.

Assay of procoagulant activity. PCA was quantitated with a one-stage recalcification assay (6). Intact viable cells were assayed to measure their surface expression of PCA. At the end of the indicated incubation times, PBM were washed once with, and resuspended in, $0.5 \mathrm{ml}$ RPMI 1640 without serum. Equal volumes of this cell suspension and pooled normal human platelet-poor plasma were incubated at $37^{\circ} \mathrm{C}$ for $1 \mathrm{~min}$. The clotting reaction was initiated with the addition of an equal volume of $25 \mathrm{mM}$ calcium chloride and time to fibrin clot formation was determined. A standard curve for milliunits of PCA was established using rabbit brain thromboplastin (Dade Division, American Hospital Supply Corporation, Miami, FL, reconstituted per manufacturer's instructions) assigned an arbitrary value of $100,000 \mathrm{mU}$. The one-stage recalcification times of sequential 10 -fold dilutions of this mixture were determined and a $\log -\log$ plot of clotting times vs. milliunits PCA was constructed. 
This plot was linear from $10 \mathrm{mU}(186 \mathrm{~s})$ to $100,000 \mathrm{mU}(16.4 \mathrm{~s})$. This standard curve was used to transform the clotting time in seconds, a nonlinear scale, to coagulant activity in milliunits, a linear scale. A log$\log$ plot of clotting times vs. 10-fold dilutions of PBM that had been incubated with LPS was also linear, indicating the standard curve was pertinent for these experiments. RPMI 1640, with or without fetal calf serum, and LPS used as stimulant consistently demonstrated no PCA $(>300$ s).

Platelet aggregation. Platelet-rich plasma was prepared from whole blood drawn from normal individuals into 0.1 vol of $3.8 \%$ trisodium citrate, $\mathrm{pH} 5.0$, and centrifuged at $150 \mathrm{~g}$ for $15 \mathrm{~min}$ at $22^{\circ} \mathrm{C}$. The plateletrich plasma was drawn off and used as below. Aggregation of platelets by mononuclear cells was found to require calcium; however, calcium added to platelet-rich plasma in the glass cuvettes of the platelet aggregometer induced platelet aggregation, presumably by activation of Factor XII and subsequent formation of thrombin. This was not seen in platelets that were washed twice in Hank's balanced salt solution without calcium or magnesium, with $1 \mathrm{mM}$ EDTA, and resuspended in Factor XIIdeficient plasma. This plasma was obtained from a local patient with severe ( $<1 \%$ of normal) congenital deficiency of coagulation Factor XII. Alternatively, before resuspension in Factor XII-deficient plasma, platelets were washed according to the method of Patscheke (16). Blood was drawn into one-seventh vol of acid citrate dextrose buffer, citric acid $0.8 \%$, sodium citrate $2.2 \%$, and dextrose $2.5 \%$. After centrifugation for 15 min at $160 \mathrm{~g}$, the platelet-rich plasma was collected, and the $\mathrm{pH}$ was adjusted to 6.5 with addition of $10 \%$ acid citrate dextrose. The platelets were then sedimented by centrifugation for $6 \mathrm{~min}$ at $480 \mathrm{~g}$, followed by washing of the platelet pellet twice in the following washing solution: $100 \mathrm{mM} \mathrm{NaCl}, 5 \mathrm{mM} \mathrm{KCl}, 2 \mathrm{mM} \mathrm{CaCl}, 1 \mathrm{mM} \mathrm{MgCl}$, $5 \mathrm{mM}$ glucose, $0.5 \mathrm{mg} / \mathrm{ml} \mathrm{BSA}, 25 \mu \mathrm{g} / \mathrm{ml}$ apyrase, $20 \mathrm{mM} \mathrm{PGE}_{1}$, and $30 \mathrm{mM}$ sodium citrate, $\mathrm{pH}$ 6.5. These platelets were also resuspended in Factor XIIdeficient plasma. Aggregation results on platelets isolated by either technique were the same. Aggregometry was carried out on an aggregometer (model 30080; Payton Scientific Inc., Buffalo, NY). The reactions were reconstituted by adding $200 \mu$ lof platelet-rich plasma $\left(4 \times 10^{8}\right.$ platelets/ $\mathrm{ml}$ in Factor XII- deficient plasma); $50 \mu \mathrm{l}$ of $16 \mathrm{mM}$ glycyl-L-prolyl-Larginyl-L-proline (Sigma Chemical Co.; included in reaction to prevent fibrin polymerization [17], final concentration $2 \mathrm{mM}$ ); $50 \mu \mathrm{l}$ of a solution of either apyrase (Sigma Chemical Co.), acetylsalicylic acid, heparin (Sigma Chemical Co.), or hirudin (Sigma Chemical Co.) at concentrations indicated in the text; $50 \mu \mathrm{l}$ of $125 \mathrm{mM}$ calcium chloride; and finally 50 $\mu$ l of mononuclear cells at $2 \times 10^{6} / \mathrm{ml}$ in RPMI 1640 . Platelets exposed to RPMI, or RPMI $+10 \mu \mathrm{g} / \mathrm{ml}$ LPS did not aggregate. To quantify the platelet aggregation response to PBM exposed to varying doses of LPS, the kinetics of platelet aggregation were quantified by calculating the slope of the initial increase in light transmission on the tracing from the platelet aggregometer (18).

For some experiments, washed platelets were resuspended in plasma obtained from an individual congenitally deficient in a single coagulation factor. Factor VII-, X-, and II-deficient plasmas were obtained commercially (George King, Overland Park, KS); Factor VIII-deficient plasma was from a local individual with severe Hemophilia A $(<1 \%$ Factor VIII activity).

Immunofluorescence. Human PBM preparations were evaluated for the presence of platelets by indirect immunofluorescence for human thrombospondin (15). PBM were allowed to adhere to poly-L-lysinecoated coverslips, fixed in $3 \%$ paraformaldehyde in $0.1 \mathrm{M}, \mathrm{pH} 7.4$ phosphate buffer. The cells were permeabilized with $-20^{\circ} \mathrm{C}$ acetone, then washed five times with Tris-buffered saline (TBS; $0.15 \mathrm{M} \mathrm{NaCl}$ and 0.01 $M$ Tris, pH 7.4). After a 30-min incubation with $3 \%$ normal sheep serum, the cells were incubated with the $\mathrm{F}\left(\mathrm{Ab}^{\prime}\right)_{2}$ of either monoclonal antithrombospondin antibody, normal mouse IgG at $2 \mu \mathrm{g} / \mathrm{ml}$, or $3 \%$ normal sheep serum for an overnight incubation at $4^{\circ} \mathrm{C}$. The coverslips were then washed five times with TBS and incubated $1 \mathrm{~h}$ at room temperature with fluorescein isothiocyanate (FITC)-conjugated sheep anti-mouse $\mathrm{F}\left(\mathrm{Ab}^{\prime}\right)_{2}$ (Sigma Chemical Co.) at $50 \mu \mathrm{g} / \mathrm{ml}$ in PBS $(0.15 \mathrm{M} \mathrm{NaCl}$ and $0.01 \mathrm{M}$ phosphate, $\mathrm{pH} 7.4$ ), $3 \%$ normal sheep serum. The cells were then washed five times with PBS and viewed for fluorescence on a Nikon type 104 inverted microscope.

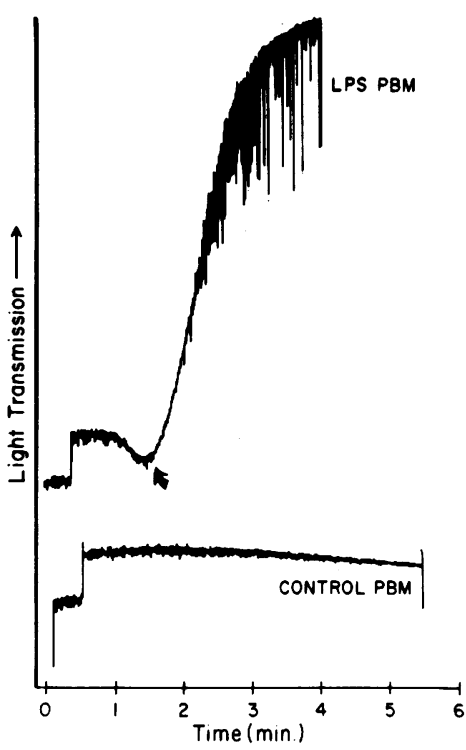

Figure 1. Peripheral blood mononuclear cells that have been exposed to LPS induce platelet aggregation.

Washed platelets were resuspended in Factor XIIdeficient plasma containing glycyl-prolyl-arginyl-proline and $\mathrm{CaCl}_{2}$. Peripheral blood mononuclear cells that had been cultured with LPS (LPS PBM) or RPMI (control PBM) were added as in Methods, and the mixture monitored for platelet aggregation. Arrow, point at which the aliquots were removed for preparation of slides, as in Figs. 5-7.

To evaluate platelet aggregates for the presence of peripheral blood monocytes, monocytes were first labeled by indirect immunofluorescence using anti-Leu-M 3 monoclonal antibody, which is specific for monocytes (reference 19; Becton Dickinson, Mountain View, CA). PBM were maintained in suspension at a concentration of $10^{6}$ cells in $50 \mu \mathrm{l}$ of RPMI. Anti-Leu-M3 or normal mouse IgG at $5 \mu \mathrm{l} / 10^{6}$ cells was added and the suspension incubated for $15 \mathrm{~min}$ at $4^{\circ} \mathrm{C}$. After being washed with RPMI, the cells were incubated with FITC sheep anti-mouse IgG or normal sheep IgG at $100 \mu \mathrm{g} / \mathrm{ml}$ in TBS, 3\% normal sheep serum for $30 \mathrm{~min}$ at $20^{\circ} \mathrm{C}$. The cells were then maintained on ice until they were added to the platelet aggregation reaction.

\section{Results}

Human platelets did not undergo aggregation when unstimulated PBM that had been cultured for $18 \mathrm{~h}$ were added to the cuvette (Fig. 1). In contrast, addition of PBM that had been incubated $18 \mathrm{~h}$ in the presence of $10 \mu \mathrm{g} / \mathrm{ml}$ of LPS brought about rapid and complete platelet aggregation (Fig. 1). The induction of platelet aggregation was dependent on the dose of LPS used to stimulate PBM, and paralleled the dose-dependent LPS induction of PCA (Fig. 2).

Because the ability of mononuclear cells to induce platelet
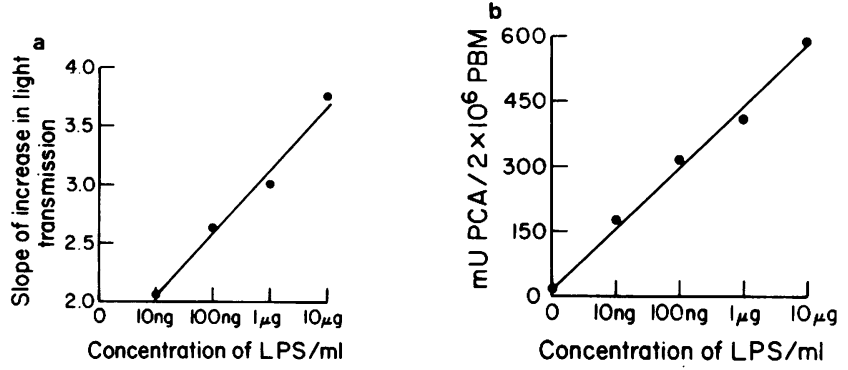

Figure 2. Dose-dependent increases in mononuclear cell PCA and kinetics of PBM induced platelet aggregation in response to LPS. (a) Kinetics of platelet aggregation: PBM that had been incubated $18 \mathrm{~h}$ with increasing doses of LPS were washed and added to Factor XII-deficient platelet rich plasma. Aggregometry was carried out as in Methods; kinetics of aggregation are expressed as the initial slope of the increase in light transmission per unit time as in reference 18. (b) Mononuclear cell PCA: PBM that had been incubated $18 \mathrm{~h}$ with increasing doses of LPS were washed and assayed for PCA as in Methods. 


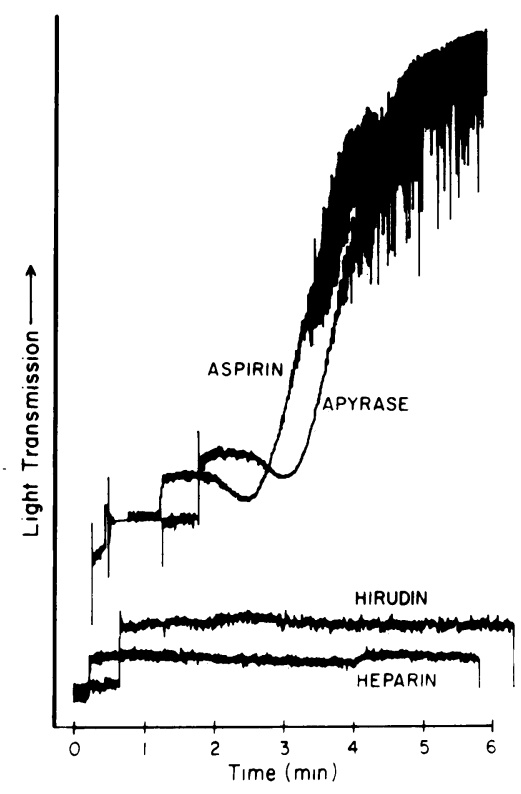

Figure 3. PBM-induced platelet aggregation appears to be dependent on thrombin. Washed platelets were resuspended in Factor XII-deficient plasma as in Fig. 1, with the addition that the platelet-rich plasma contained concentrations of aspirin, apyrase, hirudin, or heparin as specified in the text. PBM that had been cultured with LPS were added, and aggregation monitored.

aggregation seemed to correlate with surface expression of PCA, we sought evidence that thrombin, generated as a result of procoagulant expression, might mediate the platelet aggregation. Fig. 3 demonstrates that platelet aggregation induced by LPSstimulated mononuclear cells was prevented by heparinizing the plasma, or by adding hirudin. Evidence was lacking that a substance(s) other than thrombin was active in the aggregation, as neither apyrase $(25 \mu \mathrm{g} / \mathrm{ml})$ nor aspirin $(10 \mu \mathrm{g} / \mathrm{ml})$ could inhibit platelet aggregation induced by LPS-stimulated mononuclear cells (Fig. 3). Control experiments showed the expected inhibition of ADP and epinephrine-induced platelet aggregation by apyrase and aspirin.
Thrombin generation by LPS-stimulated PBM occurs by exposure of tissue factor with subsequent activation of Factors VII, X, and II (20). To test for involvement of this pathway in platelet aggregation, washed platelets were resuspended in plasmas from individuals congenitally deficient in various single clotting factors, and platelet aggregometry carried out as in Methods. Fig. 4 demonstrates poor PBM-induced aggregation in platelets suspended in Factor VII-, X-, or II-deficient plasma. The aggregation in Factor VII-deficient plasma took $>5 \mathrm{~min}$ to occur, and was probably due to Factor XII activation on the cuvette. The identical pattern was seen in Factor VII-deficient platelet-rich plasma alone, without added mononuclear cells (data not shown). The aggregation in Factor II-deficient plasma was slow, only $15 \%$ of maximum at $6 \mathrm{~min}$, and probably due to the small amount of Factor II present in the plasma $(<3 \%)$. Factor VIII-deficient plasma supported PBM-induced platelet aggregation almost as well as Factor XII-deficient plasma. These coagulation factor requirements are identical to those reported for the clotting of plasma by LPS-stimulated PBM, and are consistent with exposure of tissue factor as the initiating event culminating in thrombin generation $(5,6,10,20)$.

The possible role of monocytes in platelet aggregation was addressed using the monoclonal antibody to human monocytes, anti-Leu-M3, and indirect immunofluorescence to identify monocytes among PBM. This treatment of monocytes did not affect the amount of PCA expressed. Such labeled monocytes were added to the platelet aggregation reaction, and aliquots of the aggregation reaction were removed from the cuvette when the aggregometer showed the first signs of formation of platelet clumps (arrow, Fig. 1). Aliquots were applied to poly-L-lysinecoated glass slides, the plasma was allowed to clot (which it did in the presence of this concentration of glycyl-prolyl-arginylproline), and the gelatinous material peeled back, leaving a thin layer of individual cells, platelets, and small aggregates on the slide. The specimens were then viewed under fluorescence microscopy for the presence of fluorescent cells, monocytes, in the platelet clumps. Preliminary experiments on whole peripheral blood preparations demonstrated that indeed only monocytes were immunofluorescently labeled by this technique; platelets were not labeled at all. Fig. 5, $A$ and $B$ demonstrates a focus of a

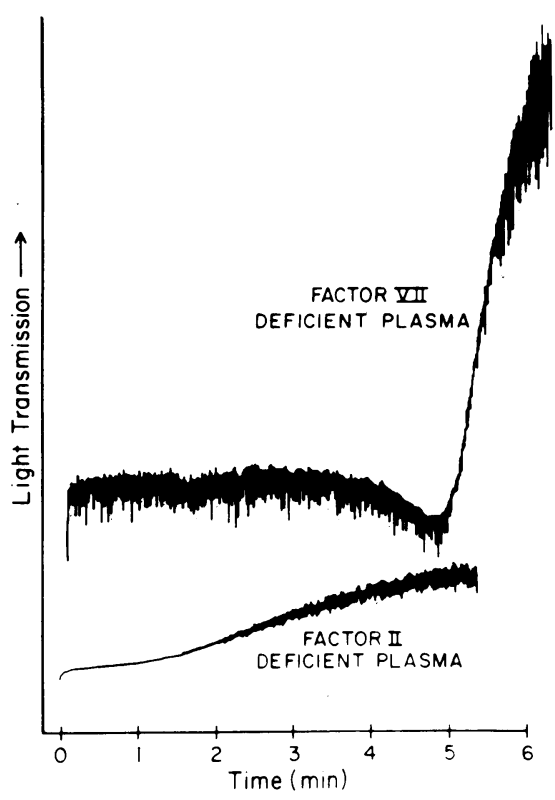

b

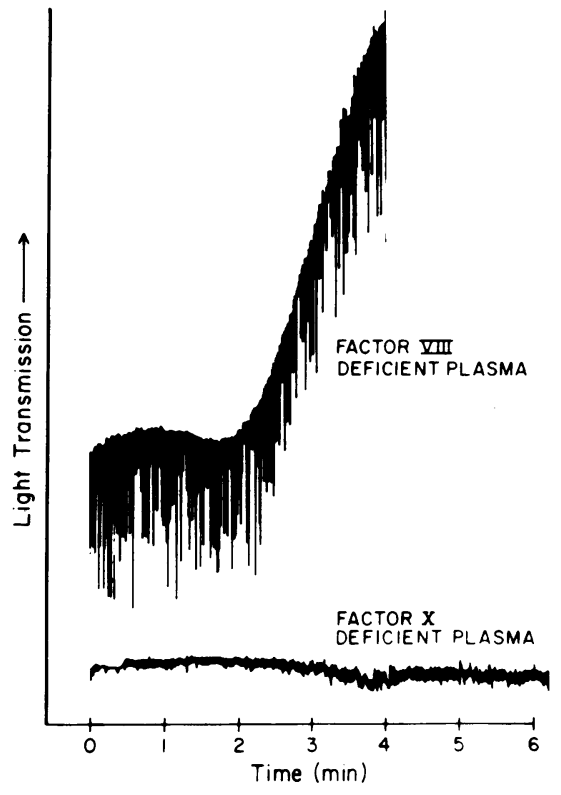

Figure 4. Coagulation factor requirements for PBM-induced platelet aggregation. Washed platelets were resuspended in plasmas from individuals congenitally deficient in single clotting factors. The plasmas contained glyclprolyl-arginyl-proline and $\mathrm{CaCl}_{2}$ as in Methods. PBM that had been cultured with LPS were added, and aggregation monitored. (a) Factor VII- and Factor II-deficient plasmas, (b) Factor VIII- and Factor X-deficient plasmas. 
platelet aggregation that contains immunofluorescently labeled monocytes. Although the focus is somewhat fuzzy due to the thickness of the preparations on the slide, the phase photomicrograph shows there is another platelet aggregate that does not contain monocytes. Of 50 aggregates examined, 32 contained monocytes. All immunofluorescently labeled monocytes were within platelet clumps; there were no free monocytes. Fig. 5, $C$ and $D$ demonstrates platelets that had been pretreated with antiLeu-M3 monoclonal antibody and FITC sheep anti-mouse IgG that were subsequently subjected to thrombin-induced platelet aggregation and processed as above. Many platelet aggregates can be seen, but there is no fluorescence. This indicates the fluorescence seen in Fig. $5 \mathrm{~B}$ is indeed due to the presence of monocytes in the platelet aggregates.

Analysis of platelet aggregation reactions wherein PBM not exposed to LPS were added to platelet-rich plasma was carried out similarly. Although there was no platelet aggregation in these reactions, an aliquot of PBM and platelets was applied to a glass slide as in the LPS-stimulated cultures. Because there was no procoagulant, the plasma did not clot, so the slides were gently washed after $10 \mathrm{~min}$. The cells and platelets on the slide were viewed by phase microscopy, and the monocytes identified as above, having been previously treated with anti-Leu-M3 and FITC sheep anti-mouse IgG. No platelet aggregates were seen, and monocytes independent of platelets were identified by morphology and fluorescence (Fig. 6, $A$ and $B$ ). The phase micrographs from this part of the experiment are noticeably sharper because the thickness of the samples from the clotted plasma was not a factor here. Fig. 6 also demonstrates that only monocytes were immunofluorescently labeled by this procedure. Specificity of labeling was demonstrated in control experiments wherein cell preparations for which nonimmune mouse IgG was substituted for anti-Leu-M3, or nonimmune sheep IgG was substituted for FITC sheep anti-mouse IgG, exhibited no fluorescence (micrographs not shown).

\section{Discussion}

DIC is marked both by fibrinogen and platelet consumption. Evidence points to monocyte procoagulant activity as an important etiologic factor in the DIC that accompanies gram-negative septicemia $(2,3,11)$. Although there have been no clinical
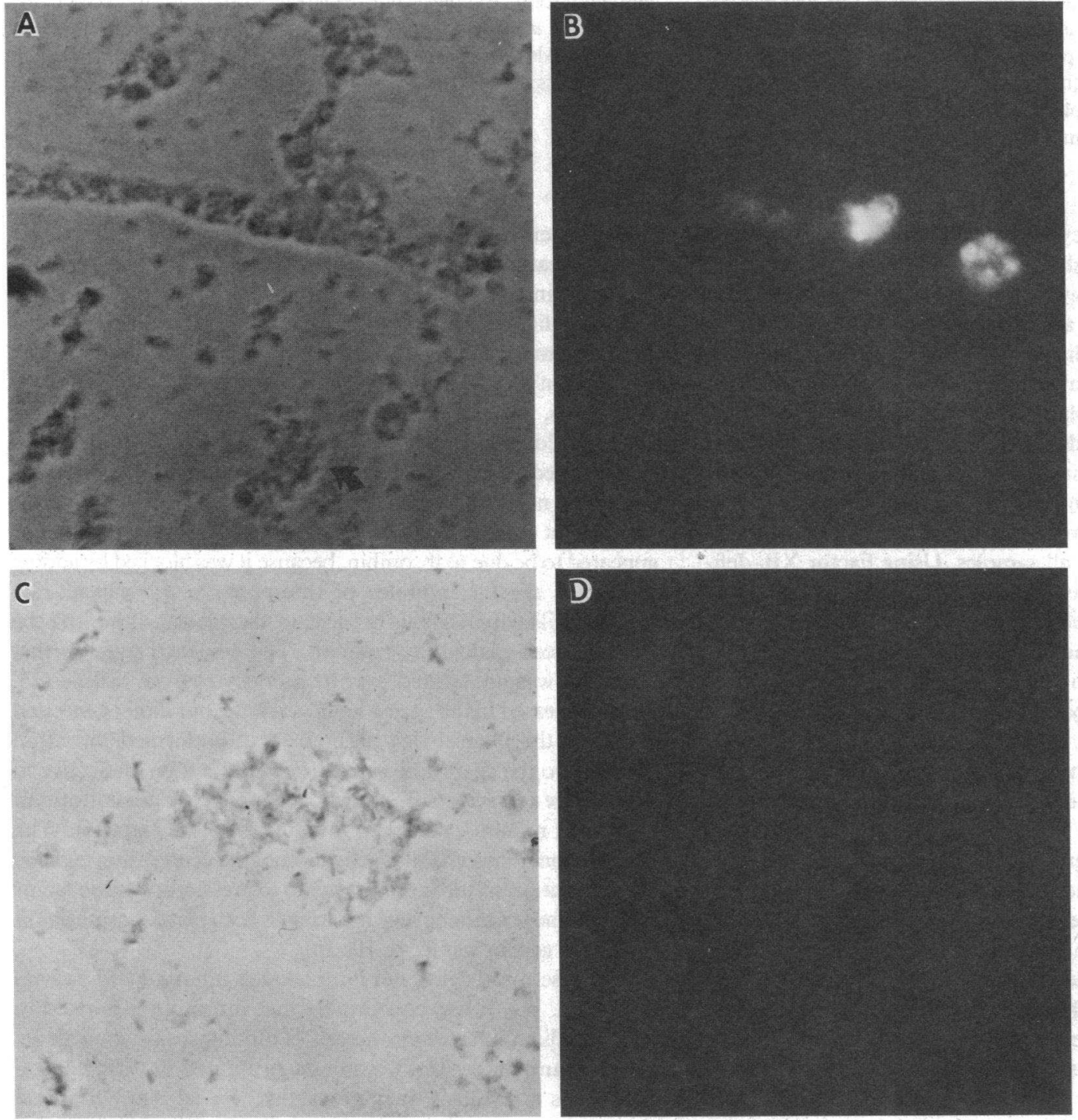

Figure 5. Identification of monocytes in the midst of platelet aggregates. Peripheral blood mononuclear cells that had been incubated with LPS were labeled using indirect immunofluorescence with anti-Leu-M3, which binds only to monocytes, then added to washed platelets resuspended in Factor XII-deficient plasma as in Methods. When the platelet aggregometer showed the very beginning of increased light transmission (aggregation), an aliquot of the mixture was removed, and placed on a glass slide. The plasma was allowed to clot, the clot peeled off, and the cells viewed by phase and fluorescence microscopy. $A$ (phase) and $B$ (fluorescence). Early aggregates of platelets and mononuclear cells. Brightly fluorescent monocytes are easily identified in the midst of a platelet clump. Arrow, a platelet clump that does not contain monocytes. There are no free monocytes. $C$ (phase) and $D$ (fluorescence). Isolated platelets were subjected to indirect immunofluorescent labeling as was done for monocytes with antiLeu-M3 antibody, then

exposed to $1.0 \mathrm{U} / \mathrm{ml}$ of thrombin in Factor XII-deficient plasma. The slide was prepared as above. No fluorescence can be seen among the platelet clumps. Magnification, 400. 


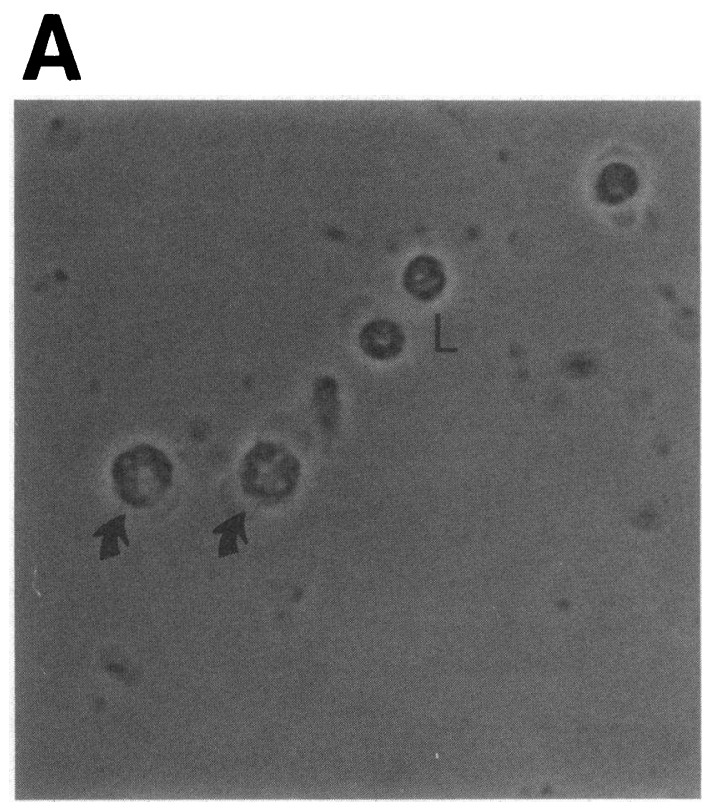

Figure 6. Monocyte-platelet aggregates do not form if monocytes do not express PCA. PBM that had not been exposed to LPS and that did not express PCA were labeled using indirect immunofluorescence and added to Factor XII-deficient platelet-rich plasma as in Fig. 5. No aggregation took place, but at a time point analagous to that in Fig. 5 , an aliquot was removed, and placed on a glass coverslip. Since there was no procoagulant the plasma did not clot, and the coverslip was

studies showing that monocyte PCA leads to platelet aggregation in this setting, it has been shown that $\beta$-thromboglobulin levels are increased in patients with DIC, a finding consistent with thrombin-induced platelet aggregation and release (21-23).

The present studies indicate that monocytes that have been induced to express PCA on their surface will cause platelet aggregation when added to platelet-rich plasma. This platelet aggregation in calcium dependent, and could best be demonstrated in Factor XII-deficient plasma. Calcium added to normal platelet-rich plasma allowed contact activation on the glass walls of the aggregometer cuvettes with subsequent thrombin generation and platelet aggregation in all samples. Using Factor XII-deficient plasma eliminated this, and allowed observation of aggregation induced by mononuclear cells. This is illustrated by the reaction carried out in Factor VII-deficient plasma (Fig. 4), wherein aggregation due to contact activation occurred $5 \mathrm{~min}$ after initiation of the incubation.

Mononuclear cells that had been incubated in the absence of LPS and then added to the platelet suspension did not induce platelet aggregation. PBM incubated with LPS expressed PCA on their surface, as detected by a one-stage plasma recalcification assay, and induced rapid aggregation of platelets. The magnitudes of both effects were dependent on the dose of LPS to which the PBM were exposed. This dependence was not due to carryover of platelets that had been incubated with the mononuclear cells and LPS, as the mononuclear cells were derived from defibrinated blood samples, and shown to be free of platelets as determined by immunofluorescence for human thrombospondin.

Platelet aggregation caused by mononuclear cells expressing PCA was not inhibited by apyrase or aspirin, indicating that neither ADP release nor cyclooxygenase activity were essential for aggregation. Platelets used in aggregation assays were washed by either of two different methods before resuspension in Factor gently washed after $10 \mathrm{~min}$. The cells were then viewed by phase $(A)$ and fluorescent $(B)$ microscopy. Monocytes can be identified by morphology $(A$, arrow) and fluorescence $(B)$; they are not part of a platelet aggregate. Also note that no other cell type is identified by anti-LeuM3; platelets do not fluoresce, nor do lymphocytes ( $L$, in $A)$. Magnification, 400.

XII-deficient plasma. Identical results were obtained in each case, indicating these findings were not a peculiarity of the isolation technique.

It is unlikely that the platelet aggregation was due to carryover of LPS from the mononuclear cell cultures. LPS-induced platelet aggregation requires the presence of aggregated IgG or immune complexes, and higher concentrations of LPS than used here (24). In addition, LPS at the concentrations used in PBM cultures, added directly to platelet-rich plasma and unstimulated PBM, did not cause aggregation.

Platelet aggregation induced by PBM that expressed PCA appeared to be due to thrombin, because it was blocked by adding hirudin, a specific inhibitor of thrombin, to the platelet-rich plasma. Likewise, adding heparin to the plasma inhibited the PCA-induced platelet aggregation. The evidence suggests that thrombin was not carried over by monocytes from culture (25, 26) and released in the aggregometry cuvette, but rather generated in situ in the platelet-rich plasma. First, preformed thrombin carried into the aggregation mixture would not be ineffective in the absence of Factor VII, X, or II. Second, preformed thrombin would not require calcium to initiate platelet aggregation. The same evidence suggests that activation of the coagulation cascade and generation of thrombin is due to expression of tissue factor on the surface of monocytes, resulting in both platelet aggregation and fibrin generation $(5,6,10,20)$.

Because monocytes, not lymphocytes among PBM express PCA $(5,6,10)$, it was conceivable that thrombin generated in the immediate microenvironment of monocytes might activate platelets, and monocytes become incorporated into platelet aggregates as a result of their proximity. We labeled peripheral blood monocytes with a specific antibody followed by a fluorescent second antibody so that we might follow the monocytes and see if they formed the nidus of platelet aggregates. These 
experiments showed that indeed monocytes were present in the middle of many platelet aggregates, although there were platelet aggregates with no monocytes present. Interestingly, there were no free monocytes seen; all were contained within platelet aggregates. We cannot discount the possibility that free monocytes were removed with the plasma clot; however, the presence of lymphocytes independent of platelet aggregates suggests that free monocytes, if present, would have been seen. It has previously been shown that $>90 \%$ of human monocytes express PCA in response to LPS (6). This suggests that most monocytes express PCA on their surface, and may be incorporated into platelet aggregates by a mechanism that is at this point unknown. It is unlikely that thrombin still bound to monocytes would induce aggregation, as bound thrombin is apparently sterically hindered from cleaving another substrate, fibrinogen (26). This proposed sequence of events is consistent with recent findings that thrombin-stimulated, but not unstimulated, human platelets selectively bind to monocytes $(27,28)$. Clinical data are also consistent with the hypothesis that monocyte induction of platelet aggregation via thrombin formation is a cause of thrombocytopenia in gram-negative septicemia. It is also provocative to hypothesize that monocyte-platelet interactions might play a role in processes such as atherosclerosis (29). This will, of course, require further study.

\section{Acknowledgements}

This work was supported by grant AI-20247 from the National Institute of Allergy and Infectious Diseases to Dr. Schwartz, who is a fellow of the John A. Hartford Foundation.

\section{References}

1. Corrigan, J. J., Jr., W. L. Ray, and N. May. 1968. Changes in the blood coagulation system associated with septicemia. N. Engl. J. Med. 279:851-856.

2. Yoshihawa, T., K. R. Tanaka, and L. B. Guze. 1971. Infection and disseminated intravascular coagulation. Medicine (Baltimore). 50: 237-258.

3. Beller, F. K. 1969. The role of endotoxin in DIC. Thromb. Diath. Haemorrh. 36(Suppl.):125-128.

4. Lerner, R. G., R. Goldstein, and G. Cummings. 1971. Stimulation of human leukocyte thromboplastic activity by endotoxin. Proc. Soc. Exp. Biol. Med. 138:145-152.

5. Edwards, R. L., F. R. Rickles, and A. M. Bobrove. 1979. Mononuclear cell tissue factor: cell of origin and requirements for activation. Blood. 54:359-370.

6. Levy, G. A., B. S. Schwartz, and T. S. Edgington. 1981. The kinetics and metabolic requirements for direct lymphocyte induction of human procoagulant monokines by bacterial lipopolysaccharide. J. Immunol. 127:357-363.

7. Thomas, L., and R. A. Good. 1952. Studies on the generalized Schwartzman reaction. I. General observations concerning the phenomenon. J. Exp. Med. 96:605-624.

8. Horn, H. G., and R. D. Collins. 1968. Studies on the pathogenesis of the generalized Schwartzman reaction: the role of granulocytes. Lab. Invest. 18:101-109.

9. Kociba, G. J., and R. A. Grieseman. 1972. Disseminated intra- vascular cogulation induced with leukocyte procoagulant. Am. J. Pathol. 69:407-416.

10. Edwards, R. L., and F. R. Rickles. 1984. Macrophage procoagulants. Progress Hemostasis Thromb. 7:183-209.

11. Hiegal, T., U. Seligsohn, E. Aghai, and M. Modan. 1978. Clinical and laboratory aspects of disseminated intravascular coagulation (DIC): a study of 118 cases. Thromb. Haemostasis. 39:122-134.

12. Ausprunk, D. H., and J. Das. 1977. Endotoxin-induced changes in human platelet membranes: morphologic evidence. Blood. 51:487496.

13. Horowitz, H. I., R. M. DesPrez, and E. W. Hook. 1962. Effects of bacterial endotoxin on rabbit platelets. II. Enhancement of platelet factor 3 activity in vitro and in vivo. J. Exp. Med. 134:642-656.

14. Levin, J., and L. E. Cluff. 1965. Platelets and the Schwartzman phenomenon. J. Exp. Med. 121:235-252.

15. Mosher, D. F., M. J. Doyle, and E. A. Jaffe. 1982. Synthesis and secretion of thrombospondin by cultured human endothelial cells. $J$. Cell Biol. 93:343-348.

16. Patscheke, H. 1981. Shape and functional properties of human platelets washed with acid citrate. Haemostasis. 10:14-27.

17. Laudano, A. P., and R. F. Doolittle. 1978. Synthetic peptide derivatives that bind to fibrinogen and prevent the polymerization of fibrin monomers. Proc. Natl. Acad. Sci. USA. 75:3085-3089.

18. Kornecki, E., G. P. Tuszynski, and S. Niewiarowski. 1983. Inhibition of fibrinogen receptor mediated platelet aggregation by heterologous anti-human platelet membrane antibody. J. Biol. Chem. 258: 9349-9356.

19. Dimitriu-Bona, A., G. R. Burmester, S. J. Waters, and R. J. Winchester. 1983. Human mononuclear phagocyte differentiation antigens. I. Patterns of antigenic expression on the surface of human monocytes and macrophages defined by monoclonal antibodies. J. Immunol. 130:145-150.

20. Tsao, B. P., D. S. Fair, L. K. Curtiss, and T. S. Edgington. 1984. Monocytes can be induced by LPS triggered $T$ lymphocytes to express functional Factor VII/VIIa protease activity. J. Exp. Med. 159:10421057.

21. Han, P., A. G. G. Turpie, and E. Genton. 1979. Plasma $\beta$-thromboglobulin: differentiation between intravascular and extravascular platelet destruction. Blood. 54:1192-1196.

22. Kaplan, K. L., and J. Owen. 1981. Plasma levels of $\beta$-thromboglobulin and platelet factor 4 as indices of platelet activation in vivo. Blood. 57:199-202.

23. Nossel, H. L., J. Wasser, K. L. Kaplan, K. S. LaGamma, I. Yudelman, and R. E. Canfield. 1979. Sequence of fibrinogen proteolysis and platelet release after intrauterine infusion of hypertonic saline. $J$. Clin. Invest. 64:1371-1378.

24. Ginsberg, M. H., and P. M. Henson. 1978. Enhancement of platelet response to immune complexes and IgG aggregates by lipid Arich bacterial lipopolysaccharides. J. Exp. Med. 147:207-218.

25. Goodnough, L. T., and H. Saito. 1982. Specific binding of thrombin by human peripheral blood monocytes. J. Lab. Clin. Med. 99: 873-884.

26. Bar-Shavit, R., A. Kahn, G. D. Wilner, and J. W. Fenton. 1983. Monocyte chemotaxis: stimulation by specific exosite region in thrombin. Science (Wash. DC). 220:728-731.

27. Jungi, T. W., M. O. Spycher, V. E. Nydegger, and S. Barandun. 1986. Platelet-leukocyte interaction: selective binding of thrombin-stimulated platelets to human monocytes, polymorphonuclear leukocytes, and related cell lines. Blood. 67:629-636.

28. Bianco, C. 1986. Monocytes recognize activated platelets through plasma membrane receptors for fibronectin. Clin. Res. 34:655A. (Abstr.)

29. Ross, R. 1986. The pathogenesis of atherosclerosis: an update. N. Engl. J. Med. 314:488-500. 